\title{
Mixed Phase Space and Tunneling
}

\author{
Arnd Bäcker, Roland Ketzmerick, Martin Richter
}

\author{
Institut für Theoretische Physik TU Dresden
}




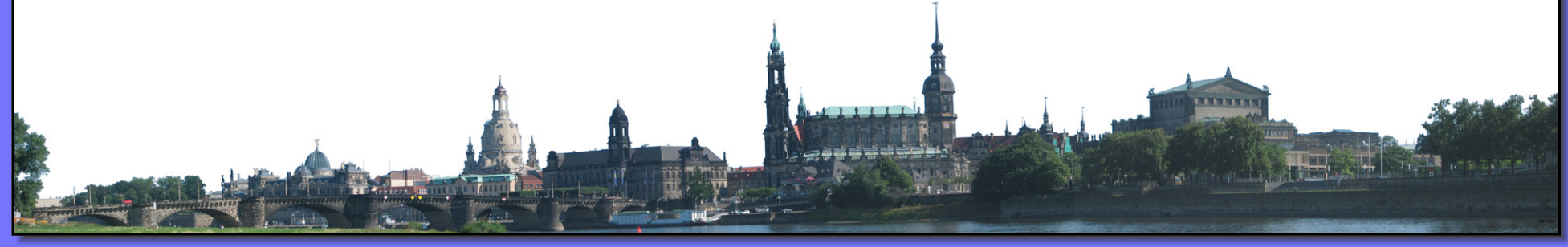

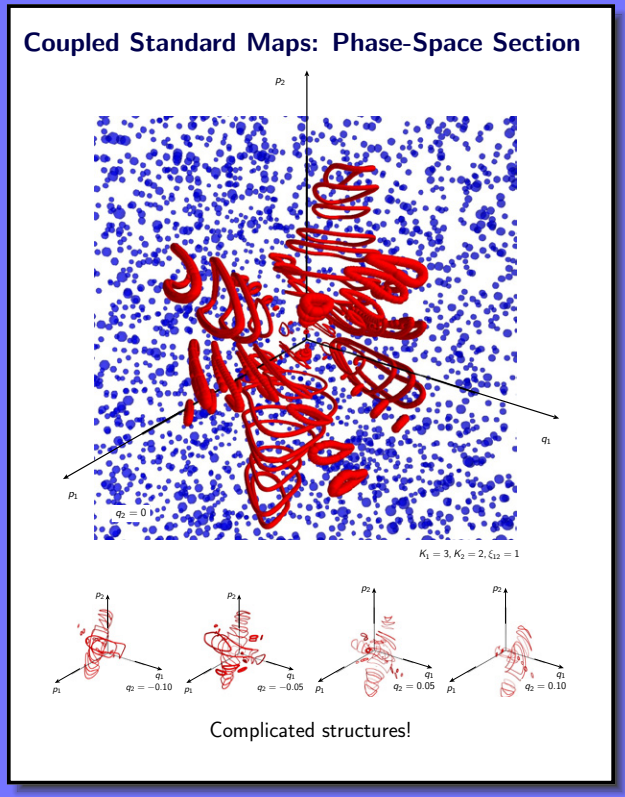

\section{Designed Map:}

Direct Regular-to-Chaotic Tunneling

Fictitious integrable system approach

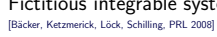

$$
\gamma_{m, n}^{d}=\|\left(1-P_{\mathrm{reg}}\right) U\left|\psi_{\mathrm{reg}}^{m, n}\right\rangle \|^{2}
$$

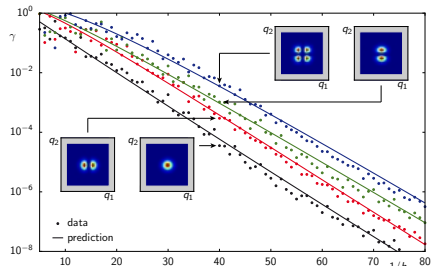

approach applicable for higher dimensional systems

Classical Description of Nonlinear Resonances $H_{\varepsilon}(\vec{I}, \vec{\vartheta}, t)=H_{\mathrm{reg}}(\vec{l}) \quad+\varepsilon V(\vec{l}, \vec{\vartheta}, t)$

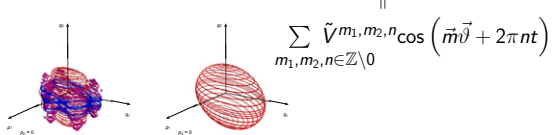

most important structure: period 5 orbit

$\rightarrow 5: 0: 1$ and $-1: 1: 0$ resonance

$\rightarrow$ also $5 \cdot \alpha-\beta: \beta: \alpha$ resonances for all $\alpha, \beta \in \mathbb{Z} \backslash 0$

after averaging over fast degrees of freedom:

$H_{\varepsilon}(\vec{J}, \vec{\theta})=\tilde{H}_{\text {reg }}(\vec{J})+\varepsilon \sum_{\alpha, \beta \in \mathbb{Z} \backslash 0} \tilde{V}^{5 \cdot \alpha-\beta, \beta, \alpha} \cos \left((5 \cdot \alpha-\beta) \theta_{1}+\beta \theta_{2}\right)$ aim: read from classical phase space
Designing a Model with Clean Phase Space

- Hamiltonian:

- Map:

$$
\begin{aligned}
H=T(\vec{p})+\sum_{n \in \mathbb{Z}} \delta(t-n) V(\vec{q}) & \left(\begin{array}{c}
p_{1}^{\prime} \\
p_{2}^{\prime} \\
q_{1}^{\prime} \\
q_{2}^{\prime}
\end{array}\right)=\left(\begin{array}{c}
p_{1}-\frac{\partial V}{\partial q_{1}}\left(q_{1}^{\prime}\right) \\
p_{2}-\frac{\partial \vartheta}{\partial q_{2}}\left(q_{2}^{\prime}\right) \\
q_{1}+p_{1} \\
q_{2}+p_{2}
\end{array}\right) \\
\text { with } T(\vec{p})=\frac{p_{1}^{2}}{2}+\frac{p_{2}^{2}}{2} & \text { with periodic boundaries }
\end{aligned}
$$

- Coupled standard maps:

$$
\begin{aligned}
& V(\vec{q})= \\
& \frac{K_{1}}{4 \pi^{2}} \cos \left(2 \pi q_{1}\right)+\frac{K_{2}}{4 \pi^{2}} \cos \left(2 \pi q_{2}\right) \\
& \\
& +\frac{\xi_{12}}{4 \pi^{2}} \cos \left(2 \pi\left(q_{1}+q_{2}\right)\right)
\end{aligned}
$$

- Designed map:
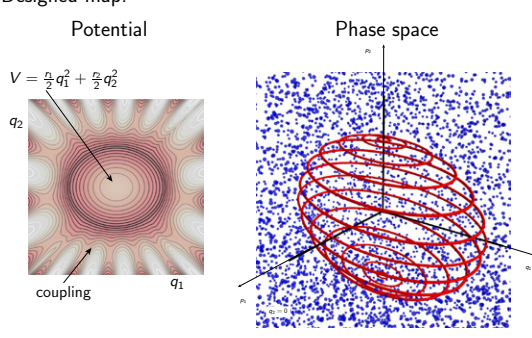

Designed Map:

Nonlinear Resonances

$$
\begin{aligned}
V\left(q_{1}, q_{2}\right)= & \frac{r_{1}}{2} q_{1}^{2}+\frac{r_{2}}{2} q_{2}^{2}+ \\
& \frac{R_{1}}{3} q_{1}^{3}+\frac{R_{2}}{3} q_{2}^{3}+ \\
& R_{12} q_{1}^{2} q_{2}
\end{aligned}
$$

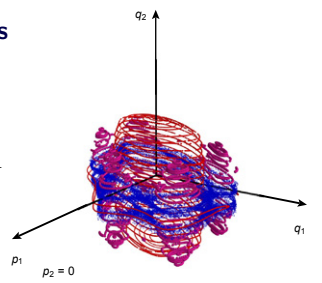

Frequency Map
[Laskar, tcarus 1990, Laskar et at., Physica D 1992]

important resonances

$m_{1} \omega_{1}+m_{2} \omega_{2}=n$ :

$5 \omega_{1}+0 \omega_{2}=1$

$-\omega_{1}+\omega_{2}=0$

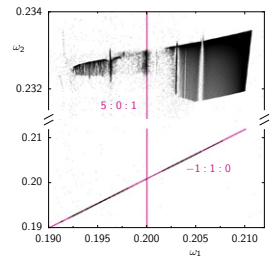

\section{Perturbation Theory}

What influences quasi modes $\left|\psi_{\mathrm{reg}}^{m, n}\right\rangle$ ?

$$
\begin{aligned}
& \left|\tilde{\psi}_{\mathrm{reg}}^{m, n}\right\rangle=\left|\psi_{\mathrm{reg}}^{m, n}\right\rangle+\sum_{k, l \neq m, n} \frac{\left\langle\psi_{\mathrm{reg}}^{k, l}|V| \psi_{\mathrm{reg}}^{m, n}\right\rangle}{E_{m, n}-E_{k, l}}\left|\psi_{\mathrm{reg}}^{k, l}\right\rangle+
\end{aligned}
$$

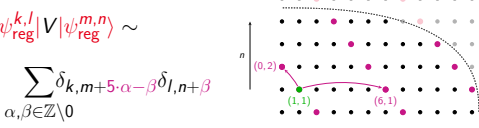

Understanding Peak Positions

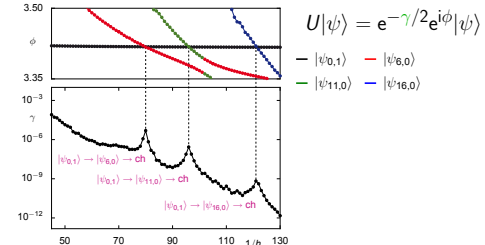

Quantum Mechanics $U|\psi\rangle=\mathbf{e}^{2 \pi \mathbf{i} \phi}|\psi\rangle$

Time evolution operator
$U=\mathrm{e}^{-i V / \hbar_{\text {eff }}} \mathrm{e}^{-i T / \hbar_{\text {eff }}}$

with $h_{\text {eff }}=1 / 40$

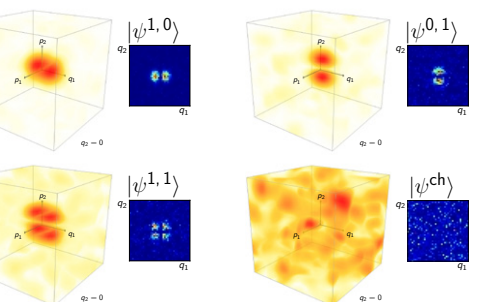

Open System

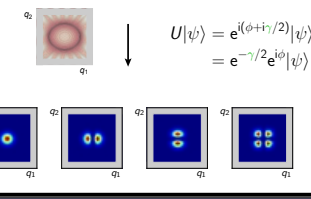

Designed Map

Tunneling Rates \& Nonlinear Resonances

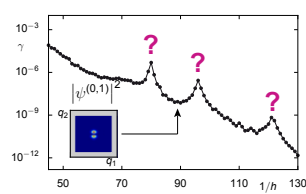

For 2D maps:

- Resonance-assisted tunneling

[Brodier, Schlagheck, Ullmo PRL 2001]
[Wimberger, Schlagheck, Eltschla, Buchleitner PRL 2006]

- Combination with Direct-regular-to-chaotic tunneling

[Lök, Bächer, Ketzmerick, Schlaghheck, PRL 2010]

$\gamma_{m}=\|\left(1-P_{\text {reg }}\right) U\left|\tilde{\psi}_{\text {reg }}^{m, n}\right\rangle \|^{2}$

$\begin{aligned} \gamma_{m} & \left.=\|\left(1-P_{\text {reg }}\right) U \psi_{\text {reg }}^{m, r}\right\rangle \|^{2} \\ \left.\gamma_{\text {reg }}^{m}\right\rangle & =\left|\psi_{\text {reg }}^{m}\right\rangle+\frac{V_{r: s}}{E_{m}-E_{m+r}}\left|\psi_{\text {reg }}^{m+r}\right\rangle+\frac{V_{r: s}}{E_{m}-E_{m+r}} \frac{V_{r: s}}{E_{m}-E_{m+2 r}}\left|\psi_{\text {reg }}^{m+2}\right\rangle+V_{r s}\end{aligned}$

$\gamma_{m}=\gamma_{m}^{d}+\left|\frac{V_{r: s}}{E_{m}-E_{m+r}}\right|^{2} \gamma_{m+r}^{d}+\left|\frac{V_{r \cdot s}}{E_{m}-E_{m+r}} \frac{V_{r \cdot s}}{E_{m}-E_{m+2 r}}\right|^{2} \gamma_{m+2 r}^{d}+$.

\section{Outlook}

- Direct regular-to-chaotic tunneling:

- apply fictitious-integrable-system approach

to generic systems $-P_{\text {reg, }}\left|\psi^{\text {reg }}\right\rangle$ ?

- Resonance-assisted tunneling:

- Quantitative understanding

- Quantitative understanding
$\left(\tilde{V}^{5 \cdot \alpha-\beta, \beta, \alpha}\right.$ from classical phase space)

- Presence of multiple resonances separated in phase space

- Influences of non-barrierness of tori

(Arnol'd-diffusion-like behaviour)?

- Role of partial barriers?

- Influences of different types of fixed points? 